Article

\title{
CaCML13 Acts Positively in Pepper Immunity Against Ralstonia solanacearum Infection Forming Feedback Loop with CabZIP63
}

\author{
Lei Shen ${ }^{1,2,3}$, Sheng Yang ${ }^{1,2,3}$, Deyi Guan ${ }^{1,2,3}$ and Shuilin $\mathrm{He}^{1,2,3, *}$ \\ 1 National Education Ministry Key Laboratory of Plant Genetic Improvement and Comprehensive Utilization, \\ Fujian Agriculture and Forestry University, Fuzhou 350002, China; shorttubelycoris07@163.com (L.S.); \\ yangsheng2061@163.com (S.Y.); gdyfujian@126.com (D.G.) \\ 2 Key Laboratory of Applied Genetics of universities in Fujian Province, Fujian Agriculture and Forestry \\ University, Fuzhou 350002, China \\ 3 Agricultural College, Fujian Agriculture and Forestry University, Fuzhou 350002, China \\ * Correspondence: shlhe201304@aliyun.com; Tel.: +86-186-0699-8458
}

Received: 8 May 2020; Accepted: 9 June 2020; Published: 11 June 2020

\begin{abstract}
Ca}^{2+}$-signaling - which requires the presence of calcium sensors such as calmodulin (CaM) and calmodulin-like (CML) proteins-is crucial for the regulation of plant immunity against pathogen attack. However, the underlying mechanisms remain elusive, especially the roles of CMLs involved in plant immunity remains largely uninvestigated. In the present study, $\mathrm{CaCML13}$, a calmodulin-like protein of pepper that was originally found to be upregulated by Ralstonia solanacearum inoculation (RSI) in RNA-seq, was functionally characterized in immunity against RSI. CaCML13 was found to target the whole epidermal cell including plasma membrane, cytoplasm and nucleus. We also confirmed that CaCML13 was upregulated by RSI in pepper roots by quantitative real-time PCR (qRT-PCR). The silencing of CaCML13 significantly enhanced pepper plants' susceptibility to RSI accompanied with downregulation of immunity-related CaPR1, CaNPR1, CaDEF1 and CabZIP63. In contrast, $\mathrm{CaCML13}$ transient overexpression induced clear hypersensitivity-reaction (HR)-mimicked cell death and upregulation of the tested immunity-related genes. In addition, we also revealed that the G-box-containing CaCML13 promoter was bound by CabZIP63 and CaCML13 was positively regulated by CabZIP63 at transcriptional level. Our data collectively indicate that CaCML13 act as a positive regulator in pepper immunity against RSI forming a positive feedback loop with CabZIP63.
\end{abstract}

Keywords: Capsicum annuum; CaCML13; CabZIP63; immunity; Ralstonia solanacearum

\section{Introduction}

Plants are frequently exposed to potentially pathogenic microbes and have evolved a sophisticated defense system initiate defense upon pathogen attack. This process is largely regulated at the transcriptional levels by the action of different transcription factors, which themselves are activated by early stages of signaling. However, how the upstream defense-signalings are interconnected with transcription factors to activate appropriate transcription outputs upon attack of pathogens is not fully understood.

Subcellular fluctuations in $\mathrm{Ca}^{2+}$ ion concentration are among the earliest responses to pathogen attack; $\mathrm{Ca}^{2+}$-signaling is essential for plant immunity [1,2]. The $\mathrm{Ca}^{2+}$ signature is decoded and transmitted downstream by different $\mathrm{Ca}^{2+}$ sensors including calmodulin like (CML) proteins, calcium-dependent protein kinases (CDPKs) and calcineurin B-like proteins (CBLs). These $\mathrm{Ca}^{2+}$ sensors relay or decode the encoded $\mathrm{Ca}^{2+}$ signals into specific cellular and physiological responses in order to survive challenges by pathogens [3-5]. Calmodulin (CaM), which is a ubiquitous $\mathrm{Ca}^{2+}$ 
sensor in eukaryotes, plays a major role in translating these $\mathrm{Ca}^{2+}$ signatures to cellular responses. $\mathrm{CaM}$ has four $\mathrm{Ca}^{2+}$-binding EF-hand motifs. Upon binding to $\mathrm{Ca}^{2+}$, CaM undergoes a conformational change, which allows it to bind to numerous target proteins and modulate the activity of these target proteins including transcription factors involved in plant defense [6-11], as they are crucial for plant immunity, they have been found to be targeted by effectors from pathogens to repress pathogen associated molecular pattern triggered immunity (PTI) in the host plants $[12,13]$. Although CML proteins are structurally similar to calmodulin, the number of CMLs in plants is much higher than CaMs, for example, five CaMs and 32 CMLs in rice [14], four CaMs and 58 CMLs in apple [15]. CML8, CML9, CML24 in Arabidopsis thaliana act positively in immunity against Pseudomonas syringae pv. tomato DC3000 [16-19], while CML46 and CML47 in Arabidopsis thaliana act as negative regulators in immunity against Pseudomonas syringae pv maculicola [20]. A CML-interacting partner, PSEUDO-RESPONSE REGULATOR 2 (PRR2), a plant specific transcription factor has been found to act positively in salicylic acid (SA) and camalexin accumulation during plant immunity [21]. However, the role of CMLs in plant immunity are largely unknown.

Pepper (Capsicum annuum) is an agriculturally important vegetable worldwide and a Solanaceae originated from tropical and subtropical regions of Central and South America and distributed mainly in uplands in the tropical and subtropical regions with various soil-borne pathogens [22,23]. Bacterial wilt caused by infection of $R$. solanacearum is one of the most frequently occurred soil-borne diseases of pepper causing great loss in pepper production worldwide [22,24]. Our previous studies showed that CaWRKY6, CaWRKY40, CabZIP63 and CaCDPK15 act as positive regulators of pepper immunity against $R$. solanacearum infection, with CaWRKY40 being directly regulated by CaWRKY6 and CabZIP63 and indirectly by CaCDPK15, forming positive feedback loops manner [25-27]. These results indicate that $\mathrm{Ca}^{2+}$-signaling contributes to pepper immunity against $R$. solanacearum and is closely related to CabZIP63/CaWRKY40 transcriptional cascades. In this study, we report that CaCML13, a CML protein in pepper, acts as positive regulator in pepper immunity against $R$. solanacearum forming a positive feedback loop with CabZIP63.

\section{Results}

\subsection{The CML Gene Was Upregulated by R. solanacearum Inoculation}

RNA-seq data of pepper challenged with $R$. solanacearum was analyzed. A CML transcript that was found to be upregulated by $R$. solanacearum infection in roots of pepper (Figure 1a). To confirm this results, we performed another experiment by quantitative real-time PCR (qRT-PCR) to assay the transcript levels of this genes upon challenge of R. solanacearum inoculation (RSI) in the roots of pepper plants, the result showed that the transcript expression levels of this $C M L$ gene were enhanced from $3 \mathrm{~h}$ post-inoculation by RSI until $48 \mathrm{~h}$ post-inoculation with the peak observed at $12 \mathrm{~h}$ post-inoculation (Figure $1 \mathrm{~b}$ ), indicating that this $C M L$ was upregulated by RSI. 
a

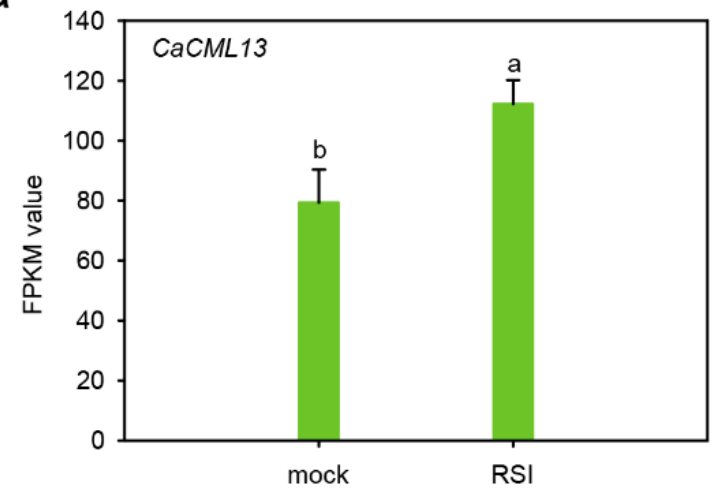

b

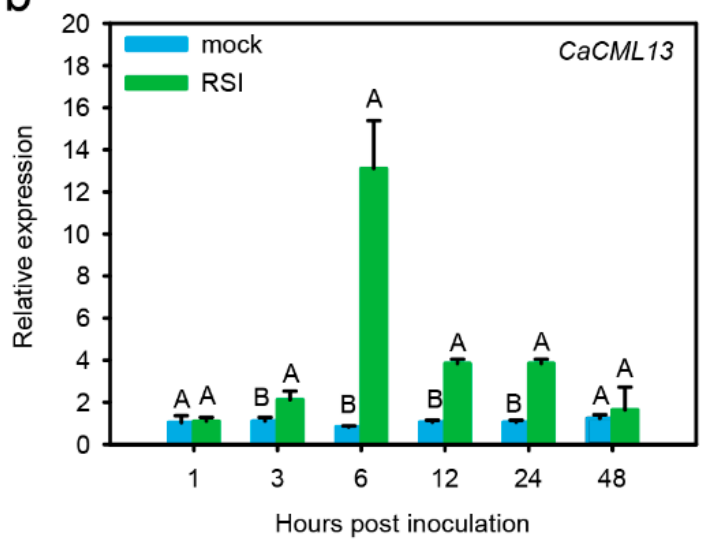

Figure 1. Transcriptional response of CaCML13 to RSI. (a) fragments per kilobase million (FPKM) of CaCML13 in pepper roots by RNA-seq. The RNA-seq data used in this study have been deposited at the China National GeneBank DataBase (CNP0001104); (b) relative transcript levels of CaCML13 in roots of pepper plants challenged with RSI by qRT-PCR. Data presented are means \pm standard error of four replicates. Different uppercase letters above the bars indicate significant differences among means $(p<0.01)$ by Fisher's protected least significant difference (LSD) test. Different lowcase letters above the bars indicate significant differences among means $(p<0.05)$ by Fisher's protected LSD test.

\subsection{The CML Is CaCML13 Exhibiting High Sequence Similarities to CMLs in Other Plant Species}

The deduced amino acid sequence of the RSI upregulated pepper CML gene, encodes a $16 \mathrm{kDa}$ protein with 444 bps, exhibited highest sequence similarities to CML13 among all of the CML members in different plant species including plants in the family Solanaceae, such as Solanum lycopersicum (CML13, XP_004234945.1) with 98\% identity, Solanum tuberosum (CML13, XP_006340368.1) with 95\% identity, Nicotiana tabacum (CML13,XP_016434602.1) with 92\% identity and other plant species including Sesamum indicum (CML13, XP_011101132.1) with 91\% identity, Ipomoea nil (CML13, XP_019192673.1) with 90\% identity, Jatropha curcas (CML13, XP_012087102.1) with 89\% identity, Citrus sinensis (CML13, XP_006477274.1) with 90\% identity and Arabidopsis thaliana (CML13, NP_172695.1) with 86\% identity (Figure 2a,b). Since it has the highest homology with AtCML13 in all CML gene family members of Arabidopsis thaliana, this gene was named CaCML13 (XP_016564798.1). Unlike CaMs that generally have four EF-hand motifs, they have only three EF-hand motifs. CaCML13 displays higher sequence similarities to its orthologs in Solanaceae than that to its orthologs in other plants including Arabidopsis thaliana (Figure 2). 
a

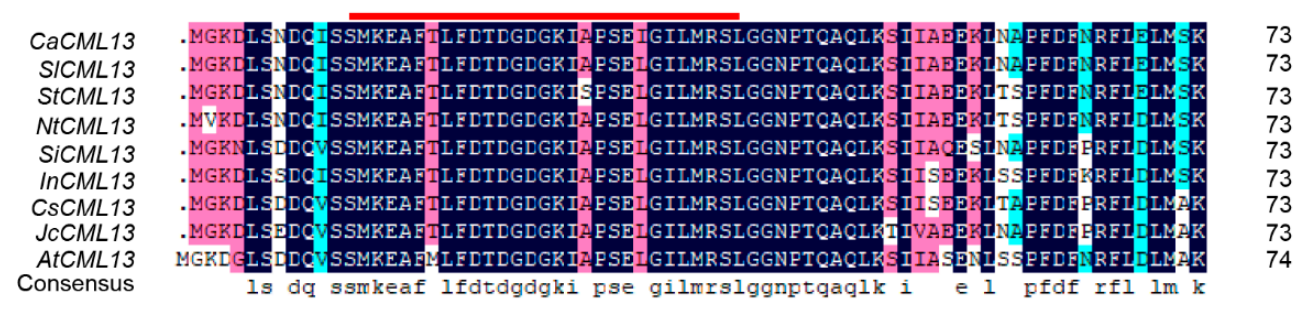

CaCML13

SICML13

StCML13

NTCML13

SiCML13

InCML13

CsCML13

JCCML13

AtCML13

Consensus
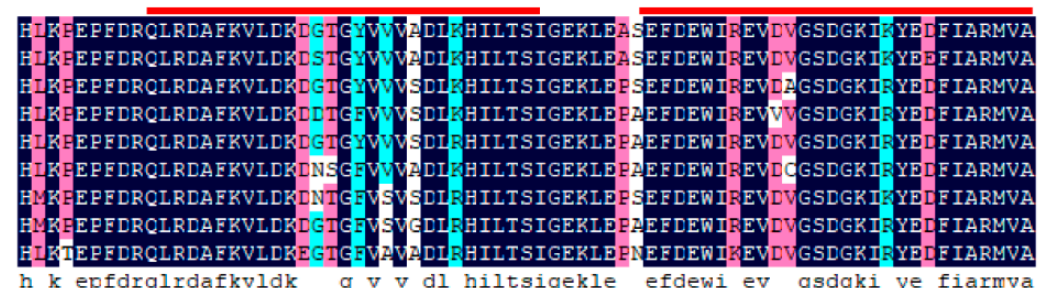

146

146

146

146
146

146

146

147

b

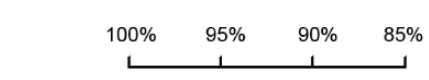

C
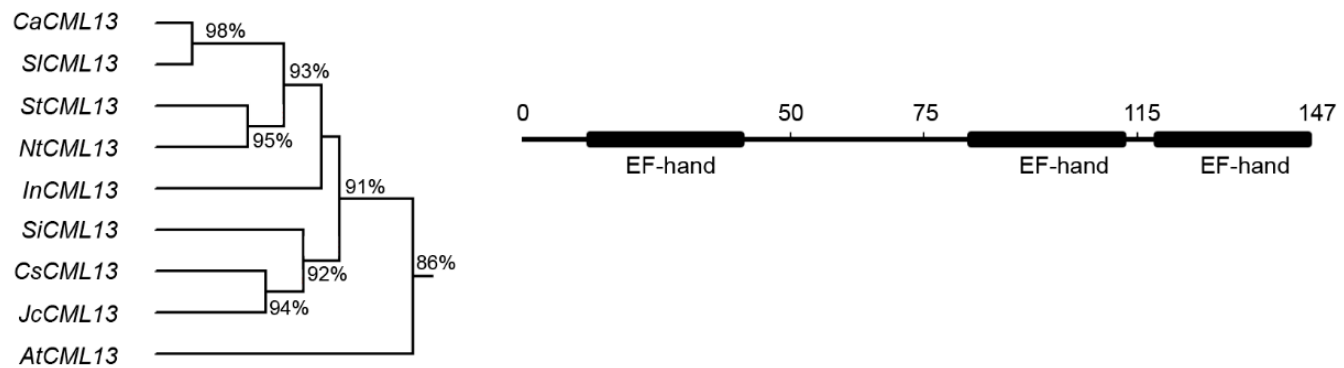

Figure 2. Deduced amino acid sequence of pepper CaCML13 and its sequence similarities to its orthologs in other plant species. (a) Comparison of deduced amino acid sequences of pepper CaCML13 to its orthologs in other plant species, amino acid residues that are conserved in at least five of the seven sequences are shaded, whereas amino acids identical in all seven proteins are shown in black, alignments were made in DNAMAN 7 (Lynnon Biosoft, USA) using the default parameters; (b) phylogenetic analysis of CaCML13 with its orthologs in other plant species including S. lycopersicum, S. tuberosum, N. tabacum, S. indicum, I. nil, J. curcas, C. sinensis and A. thaliana; (c) highly conserved EF-hand motifs in CaCML13.

\subsection{CaCML13 Is a Protein Distributes in the Whole Cell Including Plasma Membrane, Cytoplasm and the Nucleus}

To assay the subcellular targeting of CaCML13, we transient-overexpressing fused CaCML13-GFP protein by infiltration of Agrobacterium tumefaciens GV3101 cells containing 35S:CaCML13-GFP (using 35S:GFP as negative control). The GFP signals were detected at $48 \mathrm{~h}$ post-infiltration (hpi) using a confocal microscope. GFP signals within 35S:GFP-infiltrated epidermal cells of Nicotiana. benthamiana leaves were observed in plasma membrane, cytoplasm and the nucleus. Similarly, the GFP signals of CaCML13-GFP-expressed cells were also visualized in plasma membrane, cytoplasm and the nucleus (Figure 3), indicating that CaCML13 distributes in the whole cells including plasma membrane, cytoplasm and the nucleus. 


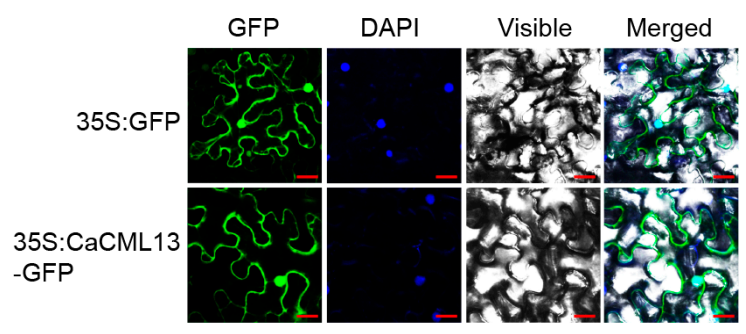

Figure 3. Subcellular localization of CaCML13 in N. benthamiana epidermal cells. N. benthamiana leaves were infiltrated with A. tumefaciens GV3101 cells harboring 35S:CaCML13-GFP (using 35S:GFP as control). Subcellular localization of the CaCML13-GFP fusion protein or GFP was viewed with a laser scanning confocal microscope at $48 \mathrm{hpi}$. The nucleus was displayed by diamidine phenyl indole (DAPI) staining, fluorescence images (GFP), bright-field images (Visible), and the corresponding overlay images (Merged) of representative cells expressing GFP or CaCML13-GFP fusion protein are shown. Bars $=25 \mu \mathrm{m}$.

\subsection{The Silencing of CaCML13 Significantly Enhanced Susceptibility to RSI}

As CaCML13 is regulated by RSI, we speculate that CaCML13 may be involved in pepper immunity against RSI. To test this possibility, we successfully and specifically silenced CaCML13 in pepper plants by an approach of virus-induced gene silencing (VIGS), displayed with significantly lower levels of CaCML13 (Figure 4a). The CaCML13 silenced pepper plants were further challenged with RSI, the result showed that $\mathrm{CaCML13}$ silenced pepper plants exhibited increased susceptibility to RSI (Figure 4b), displayed enhanced wilt symptoms and larger population of $R$. solanacearum (colony-forming units, $\mathrm{cfu}$ ) (Figure 4c), higher disease index from 5 to $14 \mathrm{dpi}$ (Figure $4 \mathrm{~d}$ ). Consistently, genes encoding pathogenesis-related proteins such as CaPR1 [28-30], CaNPR1 [31,32] and CaDEF1 [29,33] were significantly downregulated by CaCML13 silencing (Figure 4e). These results indicate that CaCML13 acts as positive regulator in pepper immunity against RSI.

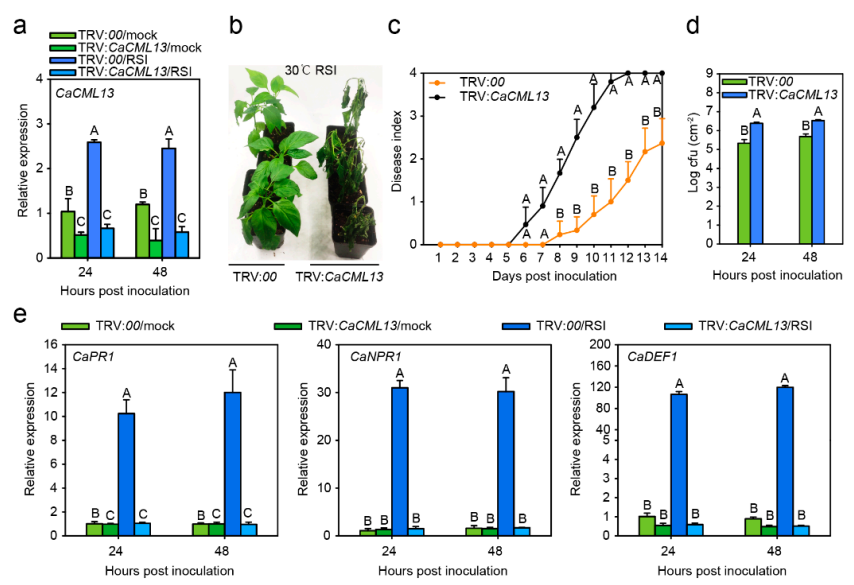

Figure 4. CaCML13-silencing-impaired pepper immunity against RSI coupled with downregulation of PR genes. (a) Test of the success of CaCML13 silencing in TRV:CaCML13 pepper plant leaves challenged with RSI at the transcriptional level at $24 \mathrm{~h}$ post-inoculation; (b) bacterial wilt symptoms in the CaCML13 silenced and the control pepper plants at 11 days post inoculation by root-irrigation; (c) disease index from 5 to 14 dpi in CaCML13 silenced pepper and the control plants challenged with RSI; (d) growth of $R$. solanacearum displayed with cfu in CaCML13 silenced and the control pepper plant leaves challenged with RSI at 24 and $48 \mathrm{~h}$ post-inoculation; (e) relative transcript levels of immunity associated genes including CaPR1, CaNPR1 and CaDEF1 were significantly downregulated by CaCML13 silencing at $24 \mathrm{~h}$ post-inoculation. In (a,c-e), data presented are means \pm standard error of four replicates. Different uppercase letters above the bars indicate significant differences among means $(p<0.01)$ by Fisher's protected LSD test. 


\subsection{Transient Overexpression of CaCML13 Triggered Intensive Hypersensitivity Reaction (HR)-Mimicked Cell Death}

To confirm the results that CaCML13 acts as positive regulator in pepper immunity, we assessed the effect of $C a C M L 13$ transient overexpression on HR-mimicked cell death and transcript expression levels of defense associated pathogenesis-related (PR) genes; the A.tumefaciens GV3101 cells harboring 35S:CaCML13 or 35S:00 (act as negative control) were infiltrated into pepper plant leaves. By analysis of qRT-PCR and western blotting with the protein isolated from CaCML13-HA transiently overexpressing in pepper leaves against anti-HA antibody, CaCML13-HA appeared to be successfully expressed in pepper leaves at transcriptional and post-translational level, respectively (Figure 5a,b). The intensive cell death was induced by infiltration of A. tumefaciens GV3101 harboring 35S:CaCML13-HA, accompanied with enhanced ion leakage displayed with high level of conductivity, darker trypan blue staining and darker diaminobenzidine (DAB) staining, indicator of $\mathrm{H}_{2} \mathrm{O}_{2}$ accumulation, while that containing 35S:00 did not trigger any cell death, clear trypan blue staining or DAB staining (Figure 5c-e).

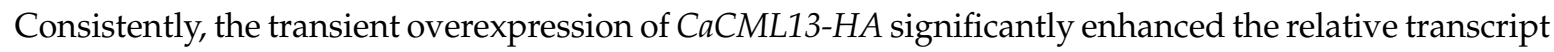
expression levels of CaPR1, CaNPR1 and CaDEF1 (Figure 5f). These results indicate that the transient overexpression of $\mathrm{CaCML13}$ significantly induced HR-mimicked cell death and immunity associated marker genes expression.
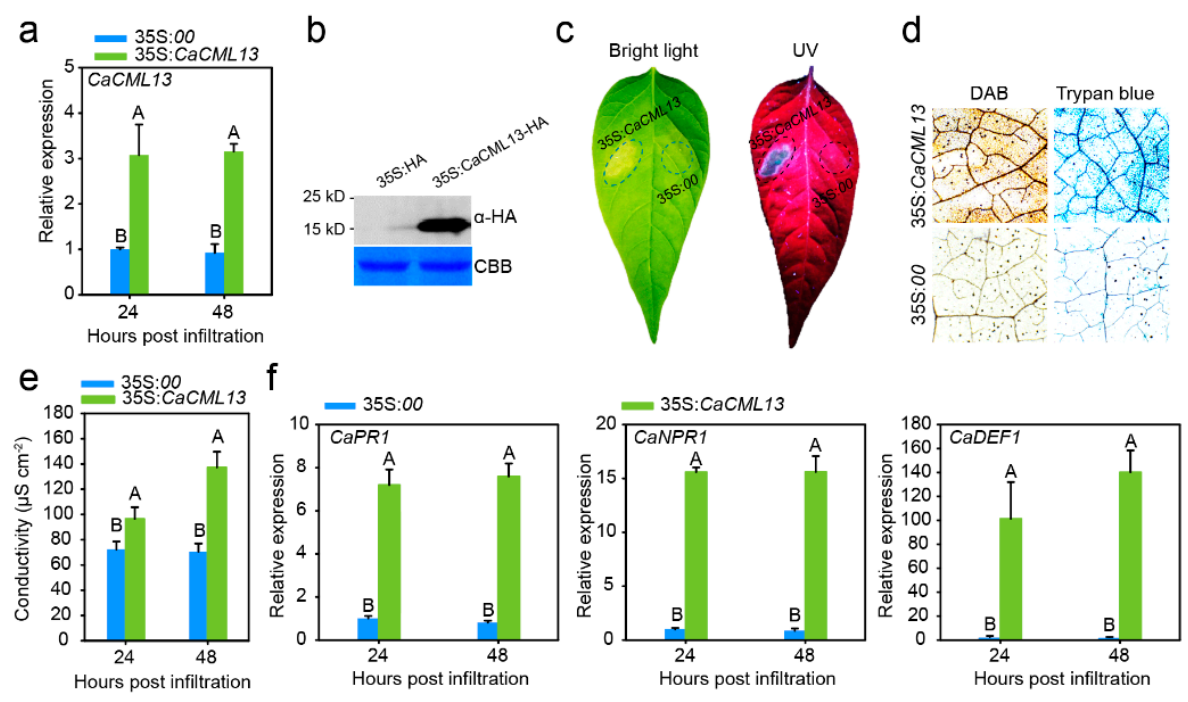

Figure 5. Effect of transient overexpression of CaCML13 on HR-mimicked cell death and expression of the tested immunity-related marker genes. $(\mathbf{a}, \mathbf{b})$ success of CaCML13 by qRT-PCR (a) and western blotting with antibody of HA (b); (c-e) HR-mimicked cell death induced by transient overexpression of CaCML13 in pepper leaves (c), HR-mimicked cell death displayed with darker trypan-blue staining and $\mathrm{H}_{2} \mathrm{O}_{2}$-accumulation manifested by darker DAB staining and (d) enhanced ion leakage displayed by high level of conductivity (e); (f) effect of CaCML13 transient overexpression on transcript levels of CaPR1, CaNPR1 and CaDEF1. In (a,e,f), data presented are means \pm standard error of four replicates. Different uppercase letters above the bars indicate significant differences among means $(p<0.01)$ by Fisher's protected LSD test.

\subsection{The G-Box-Containing CaCML13 Promoter Are Directly Bound by CabZIP63}

The predicted region about 2000 bps length acted as the promoter of CaCML13 was searched by NCBI website (https://www.ncbi.nlm.nih.gov/) using CaCML13 ORF sequence. A G-box cis-element was found in the promoter of CaCML13 (Figure 6a). This box was previously found to be bound by bZIP transcription factor [34,35]; CabZIP63 was previously found by us to be involved in pepper immunity against RSI via targeting G-box-containing-immunity-related genes [26]. We speculate that CaCML13 may be directly targeted by CabZIP63. To confirm this hypothesis, we performed a chromatin immunoprecipitation (ChIP)-PCR to test the direct binding of CabZIP63 to G-box-containing promoter 
of CaCML13. The leaves of pepper plants were infiltrated with A. tumefaciens GV3101 cells containing 35S:CaCML13-HA, which were harvested at 48 hpi for formaldehyde cross-linking, chromatins isolation, fragmentation, immunoprecipitation with antibodies of HA and DNA purification and PCR with appropriate primer pair. The result showed $C a C M L 13-H A$ was successfully expressed (Figure $6 \mathrm{~b}$ ) and a clear enrichment of CabZIP63 was found on the G-box-containing promoter fragment of CaCML13, but not in the G-box-free control promoter fragment of $\mathrm{CaCML13}$ (Figure 6c). This result indicate that CabZIP63 directly targets CaCML13.

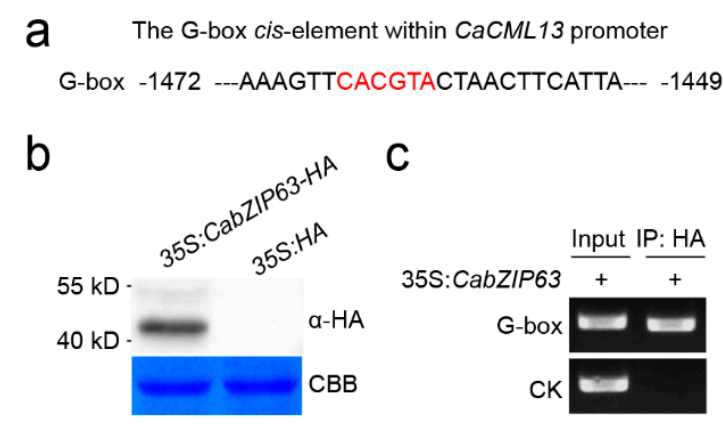

Figure 6. Enrichments of CabZIP63 on the promoter of CaCML13 by ChIP-PCR. (a) G-box-containing promoter of CaCML13 from -1472 to $-1449 \mathrm{bp}$; (b) success of CabZIP63-HA expression in pepper leaves infiltrated with $A$. tumefaciens GV30101 cells containing 35S:CabZIP63-HA by western blotting using antibodies of HA; (c) enrichments of CabZIP63 on G-box within CaCML13 promoter by ChIP-PCR. $\mathrm{CK}$ that used as a negative control represents the G-box-free DNA fragment within CaCML13 promoter. The specific primer pairs used to amplify the G-box or CK were listed in Table S1.

\subsection{The Interrelationship between CabZIP63 and CaCML13 at the Transcriptional Level}

The binding of G-box-containing CaCML13 promoter fragments by CabZIP63 indicates that CaCML13 may be regulated by CabZIP63 at the transcriptional level. To test this possibility, the relative transcript expression level of CaCML13 upon transient overexpression of CabZIP63 in pepper leaves was detected by qRT-PCR analysis. The success of CabZIP63 expression was confirmed, and the results showed that the relative transcript expression levels of $C a C M L 13$ were significantly enhanced by CabZIP63 transient overexpression (Figure 7a). In contrast, the relative transcript levels of CaCML13 were detected in the control and CabZIP63-silenced pepper leaves inoculated with $R$. solanacearum by qRT-PCR, the results showed that CabZIP63 was successful silenced and the relative transcript levels of CaCML13 were significantly downregulated by this silencing (Figure 7b). On the other hand, to investigate whether the transcript levels of CabZIP63 is regulated by CaCML13, the relative transcript levels of CabZIP63 were detected upon transient overexpression of CaCML13 or its silencing in leaves of pepper plant inoculated with $R$. solanacearum. The results showed that the relative transcript levels of CabZIP63 were significantly upregulated by transient overexpression of CaCML13, but downregulated by CaCML13 silencing (Figure 7c,d). These data suggest that CabZIP63 regulates CaCML13 transcript expression level by the targeting to CaCML13 promoter and formed a positive feedback loop with CaCML13 at transcriptional level. 
a

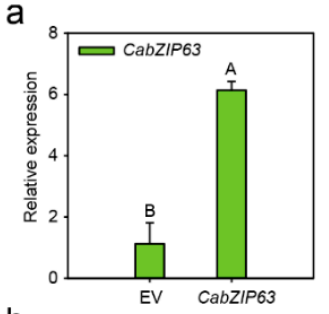

$\mathrm{b}$

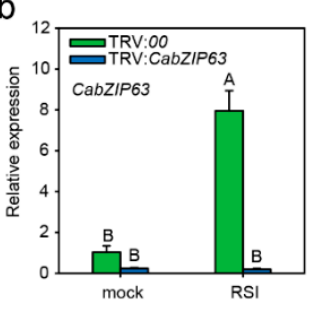

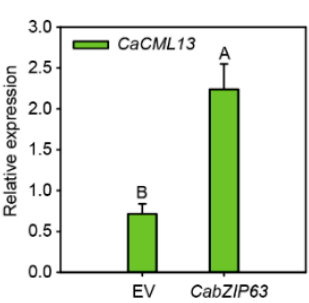

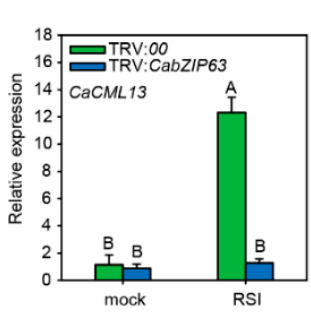

C

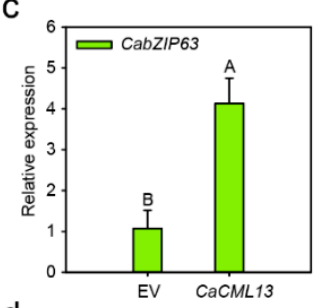

d

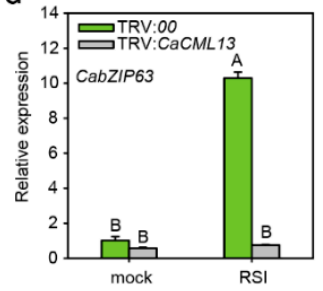

Figure 7. CabZIP63 and CaCML13 positively regulate each other at transcriptional level. (a) The relative transcript levels of CabZIP63 and CaCML13 were detected by qRT-PCR in pepper leaves upon CabZIP63 transient overexpression at $24 \mathrm{hpi}$; (b) The relative transcript levels of CabZIP63 and CaCML13 were detected by qRT-PCR in the control and CabZIP63-silenced pepper plant leaves inoculated with R. solanacearum at $24 \mathrm{~h}$ post-inoculation; (c) The relative transcript levels of CabZIP63 were detected by qRT-PCR upon transient overexpression of CaCML13 in pepper leaves at $24 \mathrm{hpi}$; (d) The relative transcript levels of CabZIP63 were detected by qRT-PCR in the control and leaves of CaCML13-silenced pepper plants inoculated with $R$. solanacearum at $24 \mathrm{~h}$ post-inoculation. Data presented are means \pm standard error of four replicates. Different uppercase letters above the bars indicate significant differences among means $(p<0.01)$ by Fisher's protected LSD test.

\section{Discussion}

$\mathrm{Ca}^{2+}$-signaling is crucial for the regulation of plant immunity against pathogen attack, the underlying mechanism remain elusive, especially the role of CMLs in plant immunity remains largely uninvestigated. In the present study, we uncover that CaCML13 is upregulated by attack of $R$. solanacearum attack and acts a positive regulator in pepper immunity against $R$. solanacearum.

A key step in both PTI and ETI is the upregulation of a multitude of defense genes, previous studies suggest that genes upregulated in host plants upon pathogen infection generally have important roles for disease resistance [25-27,36-38]. As CaCML13 was upregulated by RSI, implying its possible role in pepper immunity against $R$. solanacearum infection. This speculation was confirmed by the results that silencing of $C a C M L 13$ by VIGS significantly enhanced susceptibility of pepper plants to RSI, coupled with downregulation of immunity-related CaPR1 [28-30] and CaNPR1 [31,32], CaDEF1 [29,33] and CabZIP63 [26]. In contrast, through the transient overexpression, which were frequently employed previously [39-41], we found that CaCML13 acts positively in pepper immunity manifested by clear HR-mimicked cell death displayed enhanced ion leakage and trypan blue staining as well as $\mathrm{H}_{2} \mathrm{O}_{2}$ accumulation displayed by darker DAB staining. Since $\mathrm{H}_{2} \mathrm{O}_{2}$ accumulation was closely related to HR cell death $[42,43]$, which are generally believed to be accompanied with effector triggered immunity [44]. All these data indicate that CaCML13 acts as a positive regulator in pepper immunity against $R$. solanacearum infection. Taken together, we propose a working model that illustrate the positive feedback loop between CaCML13 and CabZIP63 in pepper response to $R$. solanacearum infection (Figure 8). CaCML13, induced by R. solanacearum infection, was targeted by CabZIP63 and formed a positive feedback loop with CabZIP63 at the transcriptional level in pepper response to $R$. solanacearum infection. 


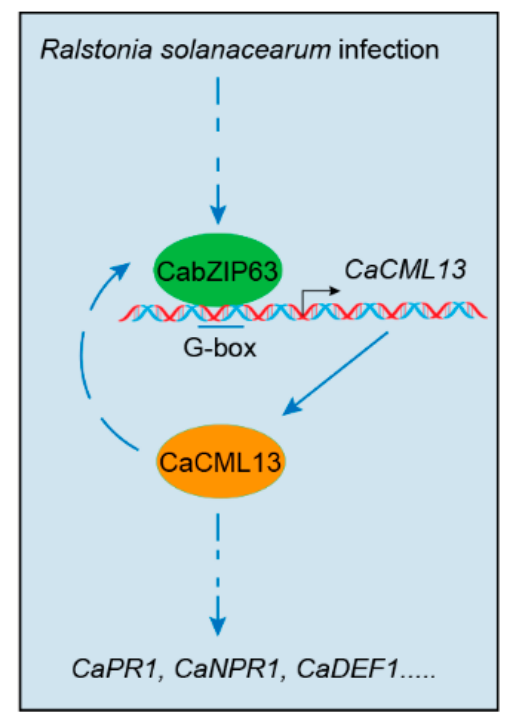

Figure 8. Proposed model of the positive feedback loop between CaCML13 and CabZIP63 in pepper response to $R$. solanacearum infection. The blue dotted arrows indicate the indirect process and the blue solid arrow indicates the direct process.

Noticeably, CabZIP63 act as positive regulator in pepper response to $R$. solanacearum infection by upregulating PR genes including CaPR1, CaNPR1, CaDEF1 and the data in the present study showed that CaCML13 positively regulates these genes, indicating that CaCML13 is related to CabZIP63 in terms of both expression and function. This speculation was further confirmed by the data that CaCML13 is directly targeted by CabZIP63 probably in G-box dependent manner and is positively regulated by CabZIP63. Given that CabZIP63 was upregulated by transient overexpression of CaCML13 and downregulated by $C a C M L 13$ silencing, it can be concluded that a positive feedback loop between CaCML13 and CabZIP63. Similar positive feedback loops were observed in the same pathosystem between CabZIP63 and CaWRKY40 [26], CaCDPK15 and CaWRKY40 [27], CaCBL1 and CaWRKY40 [45] and in other pathosystems [46,47], these positive feedback loops may be essential for immune signal amplification during plant response to pathogens. The close relationship between G-box dependent function of CabZIP63 and CaCML13 also implies the possible involvement of CaCML13 in pepper response to abiotic stresses such as heat stress and its association to ABA-signaling, as CabZIP63 has been previously found to be related to abscisic acid (ABA)-signaling-dependent CaWRKY 40 and act as positive regulator in thermotolerance [26] and G-box was believed to be a class of cis-acting elements related to ABA-signaling and bZIPs [48-50], to confirm this speculation, further investigation is required. In addition. our data also indicate that upon the activation of $\mathrm{Ca}^{2+}$ influx, multiple $\mathrm{Ca}^{2+}$ sensors may participate in the coordinated transmission of $\mathrm{Ca}^{2+}$-signaling, since CaCDPK15, CaCBL1 and CaCML13 are all participate in the pepper defense-signaling against $R$. solanacearum infection. This arrangement may make the immune-signaling less sensitive to the attacks of pathogen derived effectors, once per component is destroyed by pathogens, it may be compensated on time by other components, since components in $\mathrm{Ca}^{2+}$-signaling such as calmodulin may be target of pathogen derived effectors [12,13]. Another biologic importance of the involvement of multiple-signaling components in defense-signaling is that it can provide great regulatory potential for the feasible response of plants to different environmental conditions, since different signaling components can be modulated by internal or external stimulus.

Collectively, our data indicate that CaCML13 acts as positive regulator in pepper immunity against $R$. solanacearum infection forming a positive feedback loop with CabZIP63. Our findings imply that $\mathrm{Ca}^{2+}$-signaling mediated by multiple $\mathrm{Ca}^{2+}$ sensors including CaCML13, ABA-signaling and transcriptional cascades including CabZIP63 and CaWRKY40 are involved in pepper response to $R$. solanacearum infection and probable in thermotolerance, provide start points for further elucidation 
of mechanism underlying the crosstalk between pepper response to $R$. solanacearum infection and high temperature and high humidity in the future.

\section{Materials and Methods}

\subsection{Plant Materials and Growth Conditions}

The pepper inbred line HN42 and N. benthamiana plants were grown in sterilized soil in square plastic pots $(7 \times 7 \mathrm{~cm})$ in a growth room at $25^{\circ} \mathrm{C}$ and $60 \%$ humidity, with a light intensity of $60-70 \mu \mathrm{mol}$ photons $\mathrm{m}^{-2} \mathrm{~s}^{-1}$ in a 16-h light/8-h dark photoperiod.

\subsection{The Vectors Construction}

To construct vectors for overexpression, the full-length open reading frame (ORF) of CaCML13 or CabZIP63 were cloned into the entry vector pDONR207 by BP reaction, after confirmation by sequencing, they were further cloned into destination vectors pMDC83 and pEarleyGate201 by LR reaction, using Gateway cloning techniques (Invitrogen, Carlsbad, CA, USA). To construct vectors for gene silencing by VIGS, a specific $260 \mathrm{bp}$ fragment in the ORF of CaCML13, whose sequence specificity was confirmed by BLAST searching against genome sequence in the databases of Zunla-1 (http://peppersequence.genomics.cn/page/species/blast.jsp), were amplified by PCR with the specific primer pairs using DNA of Zunla-1 as template, and then cloned into pDONR207 by BP reaction after confirmation by sequencing, and then were further cloned into the PYL279 (TRV2) vector. All of the vectors were transformed into $A$. tumefaciens GV3101.

\subsection{VIGS Assay}

A. tumefaciens GV3101 cells harboring TRV1, TRV2:00, TRV2:CaPDS, TRV2:CaCML13 or TRV2:CabZIP63 was cultured overnight in LB media supplemented with appropriate antibiotics, then spun down and resuspended to a concentration of $\mathrm{OD}_{600}=0.8$ in the infiltration medium $(10-\mathrm{mM}$ MES, $10-\mathrm{mM} \mathrm{MgCl}_{2}, 200-\mathrm{mM}$ acetosyringone, $\left.\mathrm{pH}=5.4\right)$. The A. tumefaciens GV3101 cells containing TRV1 were mixed with cells containing TRV2:00, TRV2:CaPDS or TRV2:CaCML13 or TRV2:CabZIP63 at a 1:1 ratio and infiltrated into the cotyledons of two-week-old pepper seedlings. The plants were then placed in a growth chamber at $16^{\circ} \mathrm{C}$ in the dark for $56 \mathrm{~h}$, and then transferred to a growth room at $25^{\circ} \mathrm{C}$ and $60 \%$ humidity, with a light intensity of $60-70 \mu \mathrm{mol}$ photons $\mathrm{m}^{-2} \mathrm{~s}^{-1}$ and a 16-h light/8-h dark photoperiod, until the TRV:CaPDS plants exhibited a bleached phenotype.

\subsection{Transient Overexpression of CaCML13-HA in Pepper Leaves}

For transient expression analysis, A. tumefaciens GV3101 cells containing 35S:CaCML13-HA (using 35S:HA as control) were grown overnight and then resuspended in induction medium to $\mathrm{OD}_{600}=0.8$, approximately $100 \mu \mathrm{L}$ was infiltrated into one infiltrated site in the pepper plant leaves at the eight-leaf stage by using a syringe without a needle. The infiltrated leaves were harvested at the indicated time points for further use.

\subsection{R. solanacearum Growth and RSI}

Pepper plants or pepper leaves were inoculated with the R. solanacearum strain FJC100301 as described previously (Shen et al., 2016a; Qiu et al., 2018). R. solanacearum was grown in a SPA liquid medium $\left(200-\mathrm{g} / \mathrm{L}\right.$ potato extract, $20-\mathrm{g} / \mathrm{L}$ sucrose, $3-\mathrm{g} / \mathrm{L}$ beef extract, $5-\mathrm{g} / \mathrm{L}$ tryptone) at $28{ }^{\circ} \mathrm{C}$, then centrifuged and resuspended in a $10-\mathrm{mM} \mathrm{MgCl}_{2}$ solution to a concentration of $10^{8} \mathrm{cfu} / \mathrm{mL}$. The cells were used to inoculate pepper plants via root irrigation or pepper leaves by injection.

\subsection{Subcellular Localization}

The subcellular localization assay was performed as previously described (Shen et al., 2016a). The A. tumefaciens GV3101 cells containing 35S:CaCML13-GFP were infiltrated into N. benthamiana 
leaves, and the GFP signals were observed at 48 hpi under a laser scanning confocal microscope (TCS SP8; Leica Microsystems, Weztlar, Germany).

\subsection{ChIP Analysis}

A ChIP assay was performed as previously described [51,52]. Briefly, pepper leaves infiltrated with A. tumefaciens GV3101 cells containing 35S:HA or 35S:CabZIP63-HA were harvested at 48 hpi for chromatin isolation. The extracted chromatins were sonicated to generate DNA fragments between 200 and $500 \mathrm{bp}$ in length, which were incubated with magnetic beads (Cat\#88803, Thermo Fisher Scientific, Waltham, Massachusetts, USA) linked with anti-HA antibody for $2 \mathrm{~h}$ at $4{ }^{\circ} \mathrm{C}$, following the manufacturer's instructions. The immunoprecipitated DNA fragments were used as a template to analyze the enrichment of CabZIP63 on G-box-containing promoters of CaCML13 by using PCR with the specific primers listed in Table S1.

\section{8. qRT-PCR Assay}

To detect the transcript expression levels of the selected genes, the qRT-PCR assay was performed by using a Bio-Rad Real-Time PCR system (Bio-Rad Laboratories, California, USA) and the SYBR Premix Ex Taq II system (Takara Bio, Kyoto, Japan) with the specific primers listed in Table S1. The expression level of CaActin (GQ339766) was monitored as an internal reference gene to normalize the transcript expression levels. The Livak method was used to analyze the data [53].

\subsection{Measurement of Ion Conductivity}

Ion leakage was measured following a previously described method [26,54,55]. Leaf disks (6 mm in diameter) were taken from pepper leaves infiltrated with A. tumefaciens GV3101 cells harboring 35S:HA or 35S:CaCML13-HA.The disks were harvested at different time points and then incubated in $5 \mathrm{~mL}$ of double distilled water $\left(\mathrm{ddH}_{2} \mathrm{O}\right)$ for $1 \mathrm{~h}$ at $28^{\circ} \mathrm{C}$. The ion conductivity was measured using a Mettler Toledo 326 ion meter (Mettler Toledo, Zurich, Switzerland).

\subsection{Histochemical Staining Assay}

To assess the HR-mimicked cell death by transient overexpression of CaCML13 in pepper leaves, histochemical staining assays including trypan blue staining and DAB staining were performed as previously described $[26,27,39]$.

\subsection{Immunoblot Analysis}

The expression of CaCML13 or CabZIP63 at the post-transcriptional level was detected by western blot assay as described previous studies [45]. The pepper plant leaves infiltrated with A. tumefaciens GV3101 containing 35S:HA 35S:CaCML13-HA or 35S:CabZIP63-HA constructs were harvested in liquid nitrogen and ground into power. The total proteins were extracted by plant protein extraction buffer (25-mM Tris-HCl pH 7.5, 150-mM NaCl, 1-mM EDTA, 10\% glycerol, 1\% Triton X-100, 10-mM DTT, $1 \times$ complete protease inhibitor cocktail (Roche, Basel, Switzerland) and $2 \%(w / v)$ polyvinyl polypyrrolidone), and then incubated in ice for $1 \mathrm{~h}$. The total proteins were separated by SDS-PAGE and transmitted into polyvinylidene fluoride (PVDF) membrane at 200-mA constant current for $30 \mathrm{~min}$ by semi-dry rotary system (Bio-Rad, California, USA). The PVDF membrane was soaked in blocking buffer for $1 \mathrm{~h}$ at RT and then incubated with primary anti-HA-tag mAb antibody (Cat\#M180-3, MBL, Tokyo, Japan) at a 1:5000 dilution. Anti-IgG (H+L chain) (Mouse) pAb-HRP (Cat\#458, MBL, Tokyo, Japan) was used as a secondary antibody, diluted at 1:20,000. 


\subsection{Calculation of Disease Index of Bacterial Wilt}

Twenty R. solanacearum-inoculated pepper plants were scored every day using a disease index ranging from 0 to $4: 0$ (no wilting), 1 (1-25\% wilted), 2 (26-50\% wilted), 3 (51-75\% wilted) and 4 (76-100\% wilted or dead).

Supplementary Materials: Supplementary materials can be found at http://www.mdpi.com/1422-0067/21/11/ 4186/s1.

Author Contributions: S.H., L.S. and S.Y. conceived the research and designed the experiments. L.S. performed most the experiments with the help of S.Y., L.S., S.Y. and D.G. analyzed the data. S.H. wrote the manuscript and L.S. revised the manuscript with contributions from all authors. All authors have read and agreed to the published version of the manuscript.

Funding: This research was funded by the National Natural Science Foundation of China (31401890, 31401312), the Scientific Research Foundation of the Graduate School of Fujian Agriculture and Forestry University (324-1122yb045) and the Development Fund Project of Fujian Agriculture and Forestry University (CXZX2016158, CXZX2017548).

Acknowledgments: The authors thank Mark D. Curtis for kindly providing the Gateway destination vectors and S.P. Dinesh-Kumar of Yale University for the pTRV1 and pTRV2 vectors.

Conflicts of Interest: The authors declare no conflict of interest.

\section{References}

1. Du, L.; Ali, G.S.; Simons, K.A.; Hou, J.; Yang, T.; Reddy, A.S.; Poovaiah, B.W. Ca(2+)/calmodulin regulates salicylic-acid-mediated plant immunity. Nature 2009, 457, 1154-1158. [CrossRef] [PubMed]

2. He, Y.; Zhou, J.; Meng, X. Phosphoregulation of Ca2+ influx in plant immunity. Trends Plant Sci. 2019, 24, 1067-1069. [CrossRef] [PubMed]

3. Poovaiah, B.W.; Du, L. Calcium signaling: Decoding mechanism of calcium signatures. New Phytol. 2018, 217, 1394-1396. [CrossRef]

4. Zhang, L.; Du, L.; Poovaiah, B.W. Calcium signaling and biotic defense responses in plants. Plant Signal. Behav. 2014, 9, e973818. [CrossRef]

5. Hashimoto, K.; Kudla, J. Calcium decoding mechanisms in plants. Biochimie 2011, 93, 2054-2059. [CrossRef] [PubMed]

6. Lv, T.; Li, X.; Fan, T.; Luo, H.; Xie, C.; Zhou, Y.; Tian, C.E. The calmodulin-binding protein IQM1 interacts with CATALASE2 to affect pathogen defense. Plant Physiol. 2019, 181, 1314-1327. [CrossRef]

7. Campe, R.; Langenbach, C.; Leissing, F.; Popescu, G.V.; Popescu, S.C.; Goellner, K.; Beckers, G.J.; Conrath, U. ABC transporter PEN3/PDR8/ABCG36 interacts with calmodulin that, like PEN3, is required for Arabidopsis nonhost resistance. New Phytol. 2016, 209, 294-306. [CrossRef] [PubMed]

8. Kudla, J.; Batistic, O.; Hashimoto, K. Calcium signals: The lead currency of plant information processing. Plant Cell 2010, 22, 541-563. [CrossRef]

9. Reddy, A.S.; Ali, G.S.; Celesnik, H.; Day, I.S. Coping with stresses: Roles of calcium- and calcium/calmodulin-regulated gene expression. Plant Cell 2011, 23, 2010-2032. [CrossRef]

10. Wan, D.; Li, R.; Zou, B.; Zhang, X.; Cong, J.; Wang, R.; Xia, Y.; Li, G. Calmodulin-binding protein CBP60g is a positive regulator of both disease resistance and drought tolerance in Arabidopsis. Plant Cell Rep. 2012, 31, 1269-1281. [CrossRef]

11. Sun, T.; Huang, J.; Xu, Y.; Verma, V.; Jing, B.; Sun, Y.; Ruiz Orduna, A.; Tian, H.; Huang, X.; Xia, S.; et al. Redundant CAMTA transcription factors negatively regulate the biosynthesis of salicylic acid and N-hydroxypipecolic acid by modulating the expression of SARD1 and CBP60g. Mol. Plant 2020, 13, 144-156. [CrossRef] [PubMed]

12. Naveed, Z.A.; Bibi, S.; Ali, G.S. The phytophthora RXLR effector Avrblb2 modulates plant immunity by interfering with Ca2+ signaling pathway. Front. Plant Sci. 2019, 10, 374. [CrossRef] [PubMed]

13. Zheng, X.; Wagener, N.; McLellan, H.; Boevink, P.C.; Hua, C.; Birch, P.R.J.; Brunner, F. Phytophthora infestans RXLR effector SFI5 requires association with calmodulin for PTI/MTI suppressing activity. New Phytol. 2018, 219, 1433-1446. [CrossRef] [PubMed]

14. Boonburapong, B.; Buaboocha, T. Genome-wide identification and analyses of the rice calmodulin and related potential calcium sensor proteins. BMC Plant Biol. 2007, 7, 4. [CrossRef] 
15. Li, C.; Meng, D.; Zhang, J.; Cheng, L. Genome-wide identification and expression analysis of calmodulin and calmodulin-like genes in apple (Malusx domestica). Plant Physiol. Biochem. 2019, 139, 600-612. [CrossRef]

16. Leba, L.J.; Cheval, C.; Ortiz-Martin, I.; Ranty, B.; Beuzon, C.R.; Galaud, J.P.; Aldon, D. CML9, an Arabidopsis calmodulin-like protein, contributes to plant innate immunity through a flagellin-dependent signalling pathway. Plant J. 2012, 71, 976-989. [CrossRef]

17. Ma, W.; Smigel, A.; Tsai, Y.C.; Braam, J.; Berkowitz, G.A. Innate immunity signaling: Cytosolic Ca2+ elevation is linked to downstream nitric oxide generation through the action of calmodulin or a calmodulin-like protein. Plant Physiol. 2008, 148, 818-828. [CrossRef]

18. Zhu, X.; Perez, M.; Aldon, D.; Galaud, J.P. Respective contribution of CML8 and CML9, two arabidopsis calmodulin-like proteins, to plant stress responses. Plant Signal. Behav. 2017, 12, e1322246. [CrossRef]

19. Zhu, X.; Robe, E.; Jomat, L.; Aldon, D.; Mazars, C.; Galaud, J.P. CML8, an Arabidopsis calmodulin-like protein, plays a role in Pseudomonas syringae plant immunity. Plant Cell Physiol. 2017, 58, 307-319.

20. Chen, X.; Lu, L.; Qian, S.; Scalf, M.; Smith, L.M.; Zhong, X. Canonical and noncanonical actions of Arabidopsis histone deacetylases in ribosomal RNA processing. Plant Cell 2018, 30, 134-152. [CrossRef]

21. Cheval, C.; Perez, M.; Leba, L.J.; Ranty, B.; Perochon, A.; Reichelt, M.; Mithofer, A.; Robe, E.; Mazars, C.; Galaud, J.P.; et al. PRR2, a pseudo-response regulator, promotes salicylic acid and camalexin accumulation during plant immunity. Sci. Rep. 2017, 7, 6979. [CrossRef]

22. Hayward, A.C. Biology and epidemiology of bacterial wilt caused by pseudomonas solanacearum. Annu. Rev. Phytopathol. 1991, 29, 65-87. [CrossRef] [PubMed]

23. Ristaino, J.B.; Johnston, S.A. Ecologically based approaches to management of phytophthora blight on bell pepper. Plant Dis. 1999, 83, 1080-1089. [CrossRef] [PubMed]

24. Romero, A.M.; Kousik, C.S.; Ritchie, D.F. Temperature sensitivity of the hypersensitive response of bell pepper to Xanthomonas axonopodis pv. vesicatoria. Phytopathology 2002, 92, 197-203. [CrossRef] [PubMed]

25. Cai, H.; Yang, S.; Yan, Y.; Xiao, Z.; Cheng, J.; Wu, J.; Qiu, A.; Lai, Y.; Mou, S.; Guan, D.; et al. CaWRKY6 transcriptionally activates CaWRKY40, regulates Ralstonia solanacearum resistance, and confers high-temperature and high-humidity tolerance in pepper. J. Exp. Bot. 2015, 66, 3163-3174. [CrossRef] [PubMed]

26. Shen, L.; Liu, Z.; Yang, S.; Yang, T.; Liang, J.; Wen, J.; Liu, Y.; Li, J.; Shi, L.; Tang, Q.; et al. Pepper CabZIP63 acts as a positive regulator during Ralstonia solanacearum or high temperature-high humidity challenge in a positive feedback loop with CaWRKY40. J. Exp. Bot. 2016, 67, 2439-2451. [CrossRef]

27. Shen, L.; Yang, S.; Yang, T.; Liang, J.; Cheng, W.; Wen, J.; Liu, Y.; Li, J.; Shi, L.; Tang, Q.; et al. CaCDPK15 positively regulates pepper responses to Ralstonia solanacearum inoculation and forms a positive-feedback loop with CaWRKY40 to amplify defense signaling. Sci. Rep. 2016, 6, 22439. [CrossRef]

28. Kim, D.S.; Hwang, B.K. An important role of the pepper phenylalanine ammonia-lyase gene (PAL1) in salicylic acid-dependent signalling of the defence response to microbial pathogens. J. Exp. Bot. 2014, 65, 2295-2306. [CrossRef]

29. Kim, D.S.; Hwang, B.K. The pepper MLO gene, CaMLO2, is involved in the susceptibility cell-death response and bacterial and oomycete proliferation. Plant J. 2012, 72, 843-855. [CrossRef]

30. Kim, D.S.; Jeun, Y.; Hwang, B.K. The pepper patatin-like phospholipase CaPLP1 functions in plant cell death and defense signaling. Plant Mol. Biol. 2014, 84, 329-344. [CrossRef] [PubMed]

31. Chen, Y.Y.; Lin, Y.M.; Chao, T.C.; Wang, J.F.; Liu, A.C.; Ho, F.I.; Cheng, C.P. Virus-induced gene silencing reveals the involvement of ethylene-, salicylic acid- and mitogen-activated protein kinase-related defense pathways in the resistance of tomato to bacterial wilt. Physiol. Plant. 2009, 136, 324-335. [CrossRef] [PubMed]

32. Zhang, L.; Li, Y.; Lu, W.; Meng, F.; Wu, C.A.; Guo, X. Cotton GhMKK5 affects disease resistance, induces HR-like cell death, and reduces the tolerance to salt and drought stress in transgenic Nicotiana benthamiana. J. Exp. Bot. 2012, 63, 3935-3951. [CrossRef] [PubMed]

33. Mee Do, H.; Chul Lee, S.; Won Jung, H.; Hoon Sohn, K.; Kook Hwang, B. Differential expression and in situ localization of a pepper defensin (CADEF1) gene in response to pathogen infection, abiotic elicitors and environmental stresses in Capsicum annuum. Plant Sci. 2004, 166, 1297-1305. [CrossRef]

34. Shen, H.; Cao, K.; Wang, X. AtbZIP16 and AtbZIP68, two new members of GBFs, can interact with other G group bZIPs in Arabidopsis thaliana. BMB Rep. 2008, 41, 132-138. [CrossRef] [PubMed] 
35. Alonso, R.; Onate-Sanchez, L.; Weltmeier, F.; Ehlert, A.; Diaz, I.; Dietrich, K.; Vicente-Carbajosa, J.; Droge-Laser, W. A pivotal role of the basic leucine zipper transcription factor bZIP53 in the regulation of Arabidopsis seed maturation gene expression based on heterodimerization and protein complex formation. Plant Cell 2009, 21, 1747-1761. [CrossRef] [PubMed]

36. Ramonell, K.; Berrocal-Lobo, M.; Koh, S.; Wan, J.; Edwards, H.; Stacey, G.; Somerville, S. Loss-of-function mutations in chitin responsive genes show increased susceptibility to the powdery mildew pathogen Erysiphe cichoracearum. Plant Physiol. 2005, 138, 1027-1036. [CrossRef]

37. Bartsch, M.; Gobbato, E.; Bednarek, P.; Debey, S.; Schultze, J.L.; Bautor, J.; Parker, J.E. Salicylic acid-independent ENHANCED DISEASE SUSCEPTIBILITY1 signaling in Arabidopsis immunity and cell death is regulated by the monooxygenase FMO1 and the Nudix hydrolase NUDT7. Plant Cell 2006, 18, 1038-1051. [CrossRef]

38. Dang, F.F.; Wang, Y.N.; Yu, L.; Eulgem, T.; Lai, Y.; Liu, Z.Q.; Wang, X.; Qiu, A.L.; Zhang, T.X.; Lin, J.; et al. CaWRKY40, a WRKY protein of pepper, plays an important role in the regulation of tolerance to heat stress and resistance to Ralstonia solanacearum infection. Plant Cell Environ. 2013, 36, 757-774. [CrossRef]

39. Choi, D.S.; Hwang, I.S.; Hwang, B.K. Requirement of the cytosolic interaction between PATHOGENESIS-RELATED PROTEIN10 and LEUCINE-RICH REPEAT PROTEIN1 for cell death and defense signaling in pepper. Plant Cell 2012, 24, 1675-1690. [CrossRef]

40. Kim, N.H.; Hwang, B.K. Pepper heat shock protein 70a interacts with the type III effector AvrBsT and triggers plant cell death and immunity. Plant Physiol. 2015, 167, 307-322. [CrossRef]

41. Lee, D.H.; Choi, H.W.; Hwang, B.K. The pepper E3 ubiquitin ligase RING1 gene, CaRING1, is required for cell death and the salicylic acid-dependent defense response. Plant Physiol. 2011, 156, 2011-2025. [CrossRef] [PubMed]

42. Yoshioka, H.; Numata, N.; Nakajima, K.; Katou, S.; Kawakita, K.; Rowland, O.; Jones, J.D.; Doke, N. Nicotiana benthamiana gp91phox homologs NbrbohA and NbrbohB participate in $\mathrm{H} 2 \mathrm{O} 2$ accumulation and resistance to Phytophthora infestans. Plant Cell 2003, 15, 706-718. [CrossRef] [PubMed]

43. Do, H.M.; Hong, J.K.; Jung, H.W.; Kim, S.H.; Ham, J.H.; Hwang, B.K. Expression of peroxidase-like genes, $\mathrm{H} 2 \mathrm{O} 2$ production, and peroxidase activity during the hypersensitive response to Xanthomonas campestris pv. vesicatoria in Capsicum annuum. Mol. Plant Microbe Interact. 2003, 16, 196-205. [CrossRef] [PubMed]

44. Jones, J.D.; Dangl, J.L. The plant immune system. Nature 2006, 444, 323-329. [CrossRef]

45. Shen, L.; Yang, S.; Yang, F.; Guan, D.; He, S. CaCBL1 acts as a positive regulator in pepper response to Ralstonia solanacearum. Mol. Plant Microbe Interact. 2020. [CrossRef]

46. Liu, N.; Hake, K.; Wang, W.; Zhao, T.; Romeis, T.; Tang, D. CALCIUM-DEPENDENT PROTEIN KINASE5 associates with the truncated NLR protein TIR-NBS2 to contribute to exo70B1-mediated immunity. Plant Cell 2017, 29, 746-759. [CrossRef]

47. Wang, Y.; Schuck, S.; Wu, J.; Yang, P.; Doring, A.C.; Zeier, J.; Tsuda, K. A MPK3/6-WRKY33-ALD1-pipecolic acid regulatory loop contributes to systemic acquired resistance. Plant Cell 2018, 30, 2480-2494. [CrossRef]

48. Casaretto, J.; Ho, T.H. The transcription factors HvABI5 and HvVP1 are required for the abscisic acid induction of gene expression in barley aleurone cells. Plant Cell 2003, 15, 271-284. [CrossRef]

49. Kim, S.Y.; Chung, H.J.; Thomas, T.L. Isolation of a novel class of bZIP transcription factors that interact with ABA-responsive and embryo-specification elements in the Dc3 promoter using a modified yeast one-hybrid system. Plant J. 1997, 11, 1237-1251. [CrossRef]

50. Nakagawa, H.; Ohmiya, K.; Hattori, T. A rice bZIP protein, designated OSBZ8, is rapidly induced by abscisic acid. Plant J. 1996, 9, 217-227. [CrossRef]

51. Sun, T.; Zhang, Y.; Li, Y.; Zhang, Q.; Ding, Y.; Zhang, Y. ChIP-seq reveals broad roles of SARD1 and CBP60g in regulating plant immunity. Nat. Commun. 2015, 6, 10159. [CrossRef] [PubMed]

52. Qiu, A.; Lei, Y.; Yang, S.; Wu, J.; Li, J.; Bao, B.; Cai, Y.; Wang, S.; Lin, J.; Wang, Y.; et al. CaC3H14 encoding a tandem $\mathrm{CCCH}$ zinc finger protei Iis directly targeted by CaWRKY40 and positively regulates the response of pepper to inoculation by Ralstonia solanacearum. Mol. Plant Pathol. 2018, 19, 2221-2235. [CrossRef] [PubMed]

53. Livak, K.J.; Schmittgen, T.D. Analysis of relative gene expression data using real-time quantitative PCR and the 2(-Delta Delta C(T)) Method. Methods 2001, 25, 402-408. [CrossRef] 
54. Hwang, I.S.; Hwang, B.K. The pepper mannose-binding lectin gene CaMBL1 is required to regulate cell death and defense responses to microbial pathogens. Plant Physiol. 2011, 155, 447-463. [CrossRef]

55. Choi, H.W.; Lee, B.G.; Kim, N.H.; Park, Y.; Lim, C.W.; Song, H.K.; Hwang, B.K. A role for a menthone reductase in resistance against microbial pathogens in plants. Plant Physiol. 2008, 148, 383-401. [CrossRef] [PubMed] 